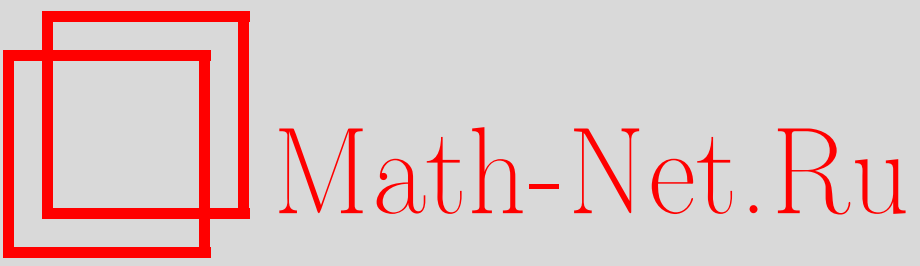

С. К. Ландо, Эквивариантно замкнутые расширения для стандартного действия ортогональной группы, УМН, 1997, том 52, выпуск 2, 171-172

DOI: https://doi.org/10.4213/rm836

Использование Общероссийского математического портала Math-Net.Ru подразумевает, что вы прочитали и согласны с пользовательским соглашением

http://www . mathnet.ru/rus/agreement

Параметры загрузки:

IP : 54.224.135.184

26 апреля 2023 г., 14:56:07 


\title{
ЭКВИВАРИАНТНО ЗАМКНУТЫЕ РАСШИРЕНИЯ ДЛЯ СТАНДАРТНОГО ДЕЙСТВИЯ ОРТОГОНАЛЬНОЙ ГРУППЫ
}

\author{
С. К. ЛАндО
}

В [2] В. Маттаи и Д. Квиллен построили представителя эквивариантного класса Тома с гауссовой плотностью относительно стандартного действия ортогональной группы на $\mathbb{R}^{2 m}$. Этот представитель может рассматриваться как функция на алгебре Ли so $(2 m)$ со значениями в модуле дифференциальных форм с гауссовой плотностью на $\mathbb{R}^{2 m}$, равная в начале координат (с точностью до несущественного в настоящий момент числового множителя) форме объема

$$
\omega_{0}=e^{-(x, x)} d x_{1} \wedge \cdots \wedge d x_{2 m}
$$

Здесь через $(\cdot, \cdot)$ обозначено скалярное произведение в $\mathbb{R}^{2 m}$. Другими словами, они построили әквивариантно замкнутое продолжсение формы объема относительно действия ортогональной группы. В настоящей заметке мы предлагаем обобщение этого резултата. А именно, пусть $P(x)=p((x, x))=p(R)$ - произвольньй многочлен, инвариантньй относительно действия ортогональной группы. Мы строим эквивариантно замкнутое расширение для формы вида

$$
P(x) \omega_{0}=e^{-(x, x)} P(x) d x_{1} \wedge \cdots \wedge d x_{2 m} .
$$

В нашей конструкции расширение выражается через итерированные интегралы.

Используя метод эквивариантной локализации (см. [1]), мы получаем равенство между итерированными интегралами и интегралом от формы (2) по всему пространству.

1. Модель Картана и эквивариантно замкнутое продолжение. Эквивариантные когомологии - это когомологии "фактормногообразия" по действию компактной группы (см. [1]). В модели Картана это когомологии следующего комплекса. Пусть $M$ - многообразие, $G$ - компактная группа, действующая на $M$ диффеоморфизмами. Пусть $\Lambda=\Lambda(M)$ - какой-нибудь комплекс дифференциальных форм на $M$, инвариантных относительно действия $G$. (Можно рассматривать различные комплексы, например, комплекс всех инвариантных форм, комплекс форм с компактным носителем, комплекс инвариантных форм с гауссовой плотностью и т.д.) Обозначим через $S(\mathbf{g})$ симметрическую алгебру алгебры Ли $\mathbf{g}$ группы $G$. Если $\varphi_{1}, \ldots, \varphi_{l} \in \mathbf{g}$ образуют базис этой алгебры Ли, то $S(\mathbf{g})$ отождествляется с кольцом многочленов от переменных $\varphi_{1}, \ldots, \varphi_{l}$.

Рассмотрим модул $\Lambda G=\Lambda G(M)=S(\mathbf{g}) \otimes \Lambda$ надколшцом $S(\mathbf{g})$. Элементы этого модуля можно считать многочленами от $\varphi_{i}$ с коэффициентами в $\Lambda$. Присваивая переменным $\varphi_{i}$ вес два, мы превращаем этот модуль в градуированньй. Дифференциал $D$ вводится формулой $D=1 \otimes(d+I)$, где $I=\sum_{k=1}^{l} i\left(X_{k}\right) \varphi_{k}$. Здесь $X_{k}$ - векторное поле на $M$, соответствующее элементу $\varphi_{k}$ алгебры Ли, а $i(\cdot)$ обозначает операцию подстановки векторного поля в дифференциальную форму.

Эквивариантные когомологии в модели Картана - это когомологии указанного комплекса.

Пусть теперь $\omega \in \Lambda$ - замкнутая дифференциальная форма, инвариантная относительно группы $G$. Мы назьваем диффференциальную форму $\Omega \in \Lambda G$ әквивариантно замкнутым продолжением формы $\omega$, если $\Omega$ замкнута относительно эквивариантного дифференциала $D$ и $\Omega(0)=\omega$, т.е. $\Omega$ совпадает с $\omega$ в нуле алгебры Ли $\mathbf{g}$.

2. Эквивариантная локализация. Эквивариантную локализацию можно понимать как способ заменить интегрирование по всему многообразию $M$ интегрированием по множеству неподвижных точек действия группы $G$ (см. [1]). Опишем вкратце процесс локализации.

Пусть $\omega_{0}-n$-форма на $M$ (где $n$ - размерность многообразия $\left.M\right)$, инвариантная относительно действия $G$. Такая форма автоматически замкнута. Обозначим через $F \subset M$ множество неподвижных точек действия группы. Предположим для простоты, что $F$ связно.

На первом шаге необходимо продолжить $\omega_{0}$ до эквивариантно замкнутой $n$-формы $\Omega$ на $M$. Такое продолжение существует не всегда.

Пусть, далее, $E$ обозначает әкв ивариантный класс Эйлера подмногообразия $F$ неподвижных точек (или, точнее, нормалњного расслоения к этому подмногообразию). Это диффференциальная форма, степень которой равна размерности нормального слоя. Теорема о локализации [1] утверждает, что в этом случае $\int_{M} \omega_{0}=\int_{F} \frac{\Omega}{E}$. Таким образом, зная эквивариантно замкнутое

Работа выполнены в институте Макса Планка, Бонн, в 1996 г. при частичной поддержке РФФИ (грант № 95-01-00846а) и INTAS (грант № 4373). 
продолжение $\Omega$ и эйлеров класс множества неподвижных точек, можно свести интегрирование по $M$ к интегрированию по $F$.

Несмотря на очевидные преимущества такого подхода, его не так-то легко воплотить в жизнь. Вычислить эйлеров класс, как правило, несложно. Однако построение эквивариантно замкнутого продолжения зачастую сопряжено со значительными трудностями. Единственный известньй автору нетривиальный пример такого построения для действия некоммутативной группы - это конструкция Маттаи-Квиллена.

3. Конструкция Маттаи-Квиллена.В простейшем некоммутативном случае стандартного действия группы $\mathrm{SO}(4)$ на $\mathbb{R}^{4}$ форма Маттаи-Квиллена выглядит следующим образом. Алгебру Ли so(4) можно отождествить с пространством кососимметрических $(4 \times 4)$-матриц. Обозначим естественные координаты в этом пространстве через $\varphi_{i j}, 1 \leqslant i<j \leqslant 4$. Тогда эквивариантно замкнутое продолжение 4-фороы $\omega=e^{-R} d x_{1} \wedge d x_{2} \wedge d x_{3} \wedge d x_{4}$, где $R=x_{1}^{2}+x_{2}^{2}+x_{3}^{2}+x_{4}^{2}$, имеет вид

$$
\begin{aligned}
\Omega= & e^{-R}\left(d x_{1} \wedge d x_{2} \wedge d x_{3} \wedge d x_{4}+\frac{1}{2}\left(\varphi_{12} d x_{3} \wedge d x_{4}-\varphi_{13} d x_{2} \wedge d x_{4}+\varphi_{14} d x_{2} \wedge d x_{3}\right.\right. \\
& \left.\left.+\varphi_{23} d x_{1} \wedge d x_{4}-\varphi_{24} d x_{1} \wedge d x_{3}+\varphi_{34} d x_{1} \wedge d x_{2}\right)+\frac{1}{4}\left(\varphi_{12} \varphi_{34}-\varphi_{13} \varphi_{24}+\varphi_{14} \varphi_{23}\right)\right) \\
= & e^{-R}\left(\omega_{0}+\omega_{1}+\omega_{2}\right),
\end{aligned}
$$

где $\omega_{j}$ - дифференциальные формы степени $4-2 j$ по переменньм $x_{i}$. В частности, эйлеров класс равен $\omega_{2}=\frac{1}{4}\left(\varphi_{12} \varphi_{34}-\varphi_{13} \varphi_{24}+\varphi_{14} \varphi_{23}\right)$.

В общем случае произвольного $k$ эквивариантно замкнутое продолжение имеет аналогичный вид:

$$
\Omega=e^{-R}\left(\omega_{0}+\omega_{1}+\cdots+\omega_{k}\right)
$$

где каждая $2(k-j)$-форма $\omega_{j}$ замкнута.

\section{4. Обобщение формулы Маттаи-Квиллена.}

Теорема. Следующая форма является әквивариантно замкнутым продолжением $2 k$-формиц $e^{-R} p(R) \omega_{0}:$

$$
\Omega_{p}=p_{0} \omega_{0}+p_{1} \omega_{1}+\cdots+p_{k} \omega_{k},
$$

где $\omega_{0}, \omega_{1}, \ldots, \omega_{k}-$ формиц, входящие в формулу Маттаи-Квиллена $(3), p_{0}(R)=e^{-R} p(R)$, $p_{1}(R)=\int_{R}^{\infty} p_{0}(\rho) d \rho, p_{j+1}(R)=\int_{R}^{\infty} p_{j}(\rho) d \rho, j=1, \ldots, k-1$.

Из принципа локализации немедленно вытекает следствие.

СлЕДСТВИЕ. Для любого многочлена $p$

$$
\int_{\mathbb{R}^{2 k}} e^{-R} p(R) \omega_{0}=\int_{0}^{\infty} \int_{\rho_{k-1}}^{\infty} \cdots \int_{\rho_{1}}^{\infty} e^{-\rho_{1}} p\left(\rho_{1}\right) d \rho_{1} \ldots d \rho_{k-1} .
$$

ДокАЗАТЕЛЬСтво теоремы. В силу того, что каждая из форм $\omega_{j}$ замкнута, эквивариантная замкнутость формы Маттаи-Квиллена означает, что $I\left(\omega_{j}\right)=d R \wedge \omega_{j+1}, j=0, \ldots, k-1$. Эквивариантный дифференциал формы (4) имеет вид

$$
\begin{aligned}
D \Omega_{p} & =d p_{0} \wedge \omega_{0}+p_{0} I\left(\omega_{0}\right)+\cdots+d p_{k} \wedge \omega_{k}+p_{k} I\left(\omega_{k}\right) \\
& =p_{0} d R \wedge \omega_{1}+d p_{1} \wedge \omega_{1}+p_{1} d R \wedge \omega_{2}+\cdots+d p_{k} \wedge \omega_{k}=0,
\end{aligned}
$$

так как $d p_{j+1}=-p_{j} d R$ в силу определения функций $p_{j}$. Теорема доказана.

\section{СПИСОК ЛИТЕРАТУРЫ}

[1] Atiyah M. F., Bott R. // Topology. 1984. V. 23. P. 1-28. [2] Matthai V., Quillen D. // Topology. 1986. V. 25. P. 85-110.

Независимый Московский университет, НИИ системных исследований РАН 